croissante des intervalles a pour effet une intensité décroissante de la formation de la matière grasse ainsi que du pourcentage du lait en matière grasse, fait auquel s'ajoute aussi l'influence de la durée du temps s'écoulant avant la traite précédente. La quantité de lait et son pourcentage en matière grasse dépendent de même, pour des intervalles égaux entre les traites, de la durée des intervalles précédents. Si la traite est faite trois fois par jour, une fois après un intervalle plus long, et deux fois après des intervalles plus courts, mais de durée égale, on obtient après l'intervalle plus court qui suit l'intervalle plus long, plus de lait et un lait plus gras qu'après l'intervalle plus court auquel précédait un intervalle de durée égale. Le pourcentage en matière grassse plus fort du lait obtenu à une certaine heure diurne pendant des traites plus nombreuses, va au préjudice du pourcentage en matière grasse du lait obtenu après l'intervalle plus long non interrompu par la traite.

Si l'on trait plus de trois fois par jour, on obtient chez les vaches qui produisent 9 kilogrammes de lait environ, plus de lait, il est vrai, mais la différence est très faible et le pourcentage en matière grasse se tient dans les limites des oscillations quotidiennes. On ne peut alors influer sur le pourcentage en matière grasse du lait par des traites plus nombreuses que dans une mesure restreinte. Si l'on trait deux fois par jour les vaches qui donnent 10 kilogrammes de lait environ, on obtient pendant l'affourragement hivernal, en administrant la ration fourragère normale à l'heure d'affourragement normale, $2 \%$ environ moins de lait et $3 \%$ environ moins de matière grasse que si l'on trait trois fois par jour.

\title{
ANALYSE BACTÉRIOLOGIQUE DES BEURRES DANS LA RÉGION DE BREST. BIOLOGIE DU BACILLE TYPHIQUE DANS LE BEURRE
}

\author{
par le Dr JEAN BRISOU
}

Médecin de la Marine, doeteur ès sciences de l'Université de Bordeaux.

Il existe des lois, décrets et définitions codifiant la vente et la fabrication du beurre en France : articles I et III, loi du 16 avril $1897 ; 2^{e}$ Congrès international pour la répression des fraudes, Paris, du 17 au 29 octobre 1909 ; loi de 1897 , modifiée le 23 juillet 1907, reprise et complétée en 1929 et 1931 ; loi du 28 février et déeret du 30 octobre 1931.

Par contre, l'étude de la pureté bactérologique de eet aliment n'a pas encore franchi les portes du laboratoire.

En 1932, Dif́nert attire l'attention de l'Académie de Médecine sur la transmission possible de la typhoïde par le beurre. Après 
analyse de 19 échantillons, il en relève 5 contaminés par le bacille paratyphique B. (3) ou le bacille d'Eberth (2).

En 1934, ZneтrI, en Italie, isole un bacille d'Eberth et 5 paratyphiques B. de 36 beurres examinés.

Dans la région brestoise, au cours de l'hiver 1934-1935, nous avons repris quelques expériences sur cette question que nous allons maintenant exposer.

\section{TECHNIQUE}

Nous avons déjà exposé à la Société de Biologie une technique nouvelle d'analyse bactériologique du beurre; nous allons la rappeler ici en quelques lignes. Dans cette technique nous mettons en œuvre le pouvoir lipo-émulsionnant de la bile d'une part, et d'autre part l'action antiseptique du vert malachite sur les germes banaux. Cette action antiseptique facilite l'isolement des bacilles d'Eberth et de ses voisins.

La bile tient son pouvoir lipo-émulsionnant de ses sels biliaires et de sa mucine. Ses sels biliaires par eux-mêmes dissolvent les acides gras libres. En milieu aleoolé, $100 \mathrm{~cm}^{3}$ de bile peuvent dissoudre 19 grammes d'acides gras.

L'éloge des procédés au vert malachite n'est plus a faire. Tous les bactériologistes reconnaissent à l'heure actuelle la supériorité que ce produit mérite dans l'isolement des germes du groupe typhoparatyphique. Le lecteur désirant se documenter sur la question se reportera à notre thèse (Bordeaux, 1933).

Nous diviserons notre méthode d'analyse en 4 phases :

\section{1 er Temps: Emulsion du beurre}

Le beurre est prélevé avec un instrument métallique quelconque stérilisé ou simplement flambé. Dans un tube de gros calibre contenant $15 \mathrm{~cm}^{3}$ de bile stérilisée, nous ensemençons environ 5 grammes de beurre. Il importe que l'émulsion soit la plus homogène possible. Pour cela nous conseillons une bile très fraîche et le chauffage léger du tube sur la flamme d'un Bunsen, en frappant de petits coups très rapides l'extrémité inférieure de ce tube sur la paume de la main, en moins de 2 minutes on obtient un liquide homogène, plus ou moins opaque, jaune verdâtre. On laisse reposer quelques secondes, et l'on pratique le $2^{\text {e temps. }}$

\section{2e Temps: Ensemencement de la bile}

Dans un ballon de $200 \mathrm{~cm}^{3}$ nous mettons en présence :

$50 \mathrm{~cm}^{3}$ d'eau physiologique stérilisée ;

$50 \mathrm{~cm}^{3}$ d'eau peptonée à $6 \%$;

$2 \mathrm{~cm}^{3}$ de vert malachite à $1 / 200^{\mathrm{e}}$.

A ce mélange nous ajoutons $5 \mathrm{~cm}^{3}$ de l'émulsion bile-beurre. 
Nous prenons soin de n'ensemencer que la partie inférieure homogène du tube de bile en laissant de côté les corps gras qui n'ont pas été dissous ou émulsionnnés d'une façon stable.

L'ensemencement terminé, nous mettons notre ballon à l'étuve à $37^{\circ}$ pendant 24 ou 36 heures, quelquefois moins si le milieu est réduit trop rapidement (décoloration).

\section{3e Temps : Passage sur gélose au sous-acétate de plomb}

Nous nous inspirons toujours de la méthode de Diénert pour l'isolement des bacilles typho-paratyphiques dans les selles.

$\mathrm{Au}$ bout de 24 à 36 heures d'étuve, le milieu au vert malachite est ensemencé sur plaques de gélose au sous-acétate de plomb fraîchement préparée et coulée en boîtes de Petri.

Pour chaque analyse nous utilisons 3 plaques de milieu. Sur les trois boîtes nous ensemencons la culture au vert malachite sans rechanger l'ensemenceur. Au bout de 24 heures d'étuve, parfois un peu plus tard, apparaissent des colonies plus ou moins séparées les unes des autres suivant le numéro de la boîte. Les colonies de bacilles d'Eberth sont noires, il en est de même pour les colonies de bacille paratyphique B. Le bacille paratyphique A. et le colibacille donnent des colonies blanches. (On rencontre cependant de temps en temps quelques colibacilles producteurs $\mathrm{d}^{\prime} \mathrm{H}^{2} \mathrm{~S}$.)

\section{4 e Temps : Isolement et identification des colonies}

On prélève séparément chaque espèce de colonies et l'on procède à l'identification des germes par les méthodes habituelles : mobilité, coloration, caractères culturaux principaux, fermentation, agglutination, pouvoir pathogène s'il s'agit d'un germe suspect.

\section{Sensibilité de la technique}

Une expérimentation assez longue dont les Archives de Médecine navale donneront les détails, nous a permis d'établir la sensibilité de notre procédé d'analyse. Nous retrouverons le bacille d'Eberth dans 10 grammes de beurre souillé à raison de 100 bacilles typhiques pour 2.500.000 germes (cocci, bacilles, moisissures, etc.).

En outre, la méthode est assez rapide. En 70 heures, on isole un bacille d'Eberth d'un beurre souillé expérimentalement.

Enfin, elle est particulièrement élective. Les germes prenant le Gram sont d'emblée éliminés de la substance à analyser. Dans nos essais de souillure expérimentale de beurre par le bacille d'Eberth, ce dernier germe était le seul à pousser sur les boîtes de Petri ; tous les autres germes ayant été détruits ou inhibés par le vert malachite.

\section{RÉSULTATS}

Sur 25 beurres brestois analysés, une fois nous avons pu isoler 
un bacille présentant les caractères morphologiques, biologiques et sérologiques, du bacille d'Eberth.

Notons qu'au cours des 4 mois pendant lesquels nous avons travaillé, les typhoïdes ont été très rares à Brest.

De pareilles analyses seraient utiles en période d'épidémie, ear si la contamination du beurre atteint $4 \%$ en temps normal, il est possible qu'en période d'épidémie ce pourcentage de contamination soit plus élevé.

Rappelons qu'en 1932 Drún ERT a trouvé 6 beurres contaminés sur 19 à Paris.

\section{Vitalité du bacille typhique dans le beurre}

Nous avons abordé le problème de la vitalité du bacille d'Eberth dans le beurre sous un angle nouveau.

Jusqu'ici on considérait que la vitalité des germes devait marcher en parallèle avee l'acidité titrimétrique du beurre.

Une première expérience nous a montré que le bacille typhique vit aussi longtemps dans un beurre rance, d'acidité butyrique libre forte, que dans un beurre frais d'acidité faible.

En second lieu, à la suite de reeherches "in vitro" sur la résistance des bacilles d'Eberth à l'acide butyrique, nous avons pu montrer qu'en eau distillée le germe ne résiste pas à $0,193 \%$ d'acide butyrique libre ; alors que dans un bouillon il résiste à $0,278 \%$ et, dans un beurre, à un taux voisin de $1 \%$. Ces faits nous ont conduit à considérer la résistance du bacille d'Eberth dans le beurre non pas comme soumise aux variations d'acidité titrimétrique, mais aux variations du $p \mathrm{H}$, ou acidité ionique du milieu. Le beurre contient $8 \%$ d'eau, laquelle tient en solution des phosphates et des citrates dont le pouvoir tampon est bien connu. On note encore dans le beurre de 0,3 à $3 \%$ de caséine. On connaît le pouvoir tampon de cet amphotère (point isoélectrique $p H=4,7$ ). Il est done logique d'attribuer au beurre un pouvoir tampon suffisant pour lutter un certain temps contre la variation d'acidité due au raneissement. Ce pouvoir tampon doit maintenir le beurre aux environs de $p \mathrm{H}=$ 6,5 ; or, le bacille d'Eberth résiste jusqu'à un $p$ H de 6. Au-dessous de ce taux la vitalité du germe doit être atteinte. Un beurre, qu'il soit rance ou frais, peut done être dangereux. Il suffit que son $p H$ soit supérieur à 6,0 .

Nous pensons même qu'une chute de $p \mathrm{H}$ au-dessous de ce chiffre n'entraîne pas la mort immédiate des germes. Ceux-ci, protégés par les matières grasses, sont forcément moins vulnérables qu'en milieu aqueux.

Nous rappelons pour mémoire quelques $p H$ des produits voisins du beurre et lui donnant naissance : lait de vache, 6,61-6,67 ; 
erème, 6,48-6,62. (M M $^{\text {He }} \mathrm{V}$. Lester, Stassano et Rollet, Achard et Stassano.)

Quelques mesures effectuées sur le beurre nous ont donné des chiffres voisins. Nous basons done notre théorie sur des faits expérimentaux et sur la composition chimique du beurre.

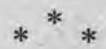

Résumons brièvement ces quelques pages:

Nous avons proposé un nouveau procédé d'analyse bactériologique du beurre. Procédé spécifique pour la recherche des germes du groupe typho-paratyphique.

Ce procédé offre une électivité et une sensibilité encourageantes. Sur 25 beurres analysés à Brest, la proportion de contamination par le bacille d'Eberth était de 4\%.

Enfin, une expérimentation biologique nous a permis de considérer la.vitalité du bacille d'Eberth dans le beurre non pas comme soumise aux variations d'acidité libre du milieu, mais soumise aux variations du $p H$ et fontions du pouvoir-tampon du beurre.

(Laboratoire de Bactériologie, Hôpital maritime de Brest.)

\title{
REVUE
}

\section{LES AGENTS DE NETTOYAGE EMPLOYÉS DANS L'INDUSTRIE LAITIERE}

\author{
par G. GÉNIN
}

Ingénieur E. P. C. I.

Les agents alcalins qui sont utilisés pour le nettoyage des récipients dans l'industrie laitière sont en nombre relativement peu élevé. Arnold H. Johnson, du Laboratoire de Recherches de la "National Dairy Products Corporation ", a étudié, dans un rapport récemment publié, la composition et l'efficacité de ces différents produits.

Les principaux agents alcalins qui sont utilisés en laiterie sont les différentes qualités, que l'on trouve sur le marché, de soude caustique, de métasilicate de sodium, de phosphate trisodique et de carbonate de soude. On trouve dans le commerce, sous des marques de fabrique les plus diverses, ces différents produits, qui sont vendus seuls ou en mélange, additionnés parfois de certains autres constituants. C'est ainsi que lorsque l'agent de nettoyage doit posséder une certaine action abrasive, on lui ajoute une faible quantité de pierre ponce ou un autre produit analogue. On peut également, pour améliorer à un certain point de vue l'efficacité de ces 